\title{
CHARACTERISATION AND STRUCTURE OF SOME NEW SMECTIC F PHASES
}

\author{
A. J. LEADBetTer, J. P. GAUGHAN, B. KeLly \\ Department of Chemistry, University of Exeter, Stocker Road, Exeter, EX4 4QD, England \\ G. W. GRAY and J. GOODBY \\ Department of Chemistry, University of Hull, Hull, HU6 7RX, England
}

\begin{abstract}
A number of new compounds of two series have been shown by miscibility studies and optical microscopy to exhibit the hitherto rare smectic $F$ phase. An example of each series has been chosen for detailed X-ray structural investigation : terephthalylidenebis-4-n-pentylaniline (TBPA) and 4-(2'-methyl butylphenyl $4^{\prime}$-n-octyloxybiphenyl-4-carboxylate) (8OSF). The structure of the $S_{F}$ phase has been determined for the first time. Locally it consists of a $C$ centred monoclinic cell with a regular hexagonal packing of the molecules in the layers. The detailed ordering is restricted to about 30 molecular diameters within a layer and to 1 or 2 layers although the gross hexagonal symmetry is preserved throughout the bulk of the sample.

The tilt direction of the hexagonal net is different for the two compounds: for TBPA it is towards an edge of the hexagon (as for the $S_{H}$ phase of TBBA) while for 8 OSF it is towards a corner. The $S_{H}$ phases of each compound have essentially the same structure but with longer range order and the two different tilt directions are maintained. Preliminary work on the $S_{\mathrm{G}}$ phases indicates that they are similar to the $S_{G}$ phase of TBBA but again with two different tilt directions.
\end{abstract}

1. Introduction. - A number of 4-(2'-methylbutyl)phenyl esters of the $4^{\prime}$-n-alk oxybiphenyl-4carboxylic acids have recently been prepared [1]. It has been shown that a number of these materials exhibit smectic $\mathbf{F}$ phases by co-miscibility experiments with the standard pyrimidine [2].

Furthermore, a number of the terephthalylidenebis-4-n-alkylanilines have also been shown to exhibit the smectic F phase [3]. These two homologous series of compounds therefore provide new $S_{F}$ materials that are readily available for study by physical methods.

In this present study one material from each series has been selected for rigorous examination by X-ray techniques, namely, terephthalylidene-bis-4-n-pentylaniline (TBPA) and 4-(2'-methylbutyl) phenyl $4{ }^{\prime}$-noctyloxybiphenyl-4-carboxylate (8OSF). The transition temperatures for these two materials are as follows :

$$
\begin{aligned}
& \mathrm{C}_{5} \mathrm{H}_{11}-\varnothing-\mathrm{N}=\mathrm{CH}-\varnothing-\mathrm{CH}=\mathrm{N}-\varnothing-\mathrm{C}_{5} \mathrm{H}_{11} \\
& \mathrm{I}-\mathrm{N}-\mathrm{S}_{\mathrm{A}}-\mathrm{S}_{\mathrm{C}}-\mathrm{S}_{\mathrm{F}}-\mathrm{S}_{\mathrm{H}}-\mathrm{S}_{\mathrm{G}} \quad \text { m.p. } 68 \\
& \begin{array}{llllll}
233 & 212 & 179 & 149 & 140 & 61
\end{array} \\
& \mathrm{C}_{8} \mathrm{H}_{17} \mathrm{O}-\varnothing-\varnothing-\mathrm{COO}-\varnothing-\mathrm{CH}_{2} \mathrm{CH}\left(\mathrm{CH}_{3}\right) \mathrm{C}_{2} \mathrm{H}_{5} \\
& \mathrm{I}-\mathrm{N}-\mathrm{S}_{\mathrm{A}}-\mathrm{S}_{\mathrm{C}}-\mathrm{S}_{\mathrm{F}}-\mathrm{S}_{\mathrm{H}}-\mathrm{S}_{\mathrm{G}} \quad \text { m.p. } 76 \\
& \begin{array}{llllll}
174 & 171 & 132 & 79 & 72 & 61
\end{array}
\end{aligned}
$$

The phases exhibited by these materials were identified by misability methods and the transition temperatures, measured by optical microscopy, were confirmed by DTA; these results will be published elsewhere. However, the microscopic textures of the smectic $F$ and smectic $G$ phases of each material as shown below. Plate 1 shows the schlieren texture of the $S_{F}$ phase of TBPA; plate 2 shows the characteristic chequerboard broken-fan texture of the $S_{F}$ phase of TBPA. These two textures are very similar to those reported by Demus et al. for the pyrimidine compound. Plate 3 shows the mosaic texture of the $S_{G}$ phase 


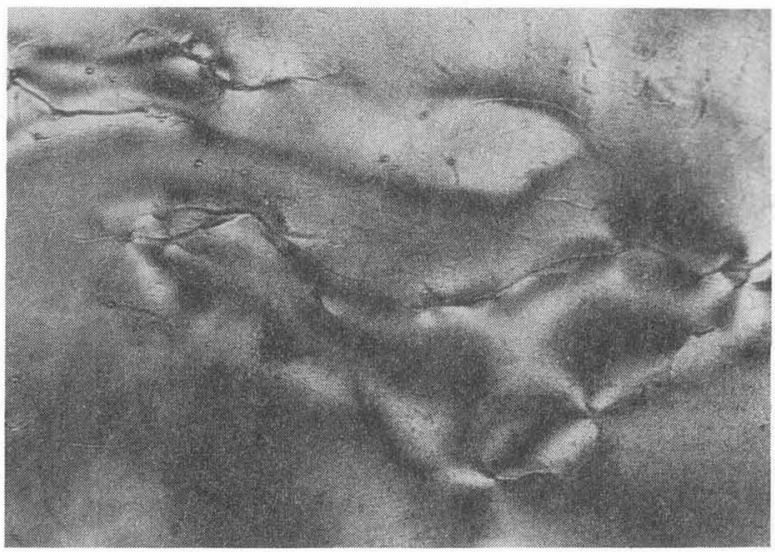

Plate 1. - The schlieren texture of the $S_{F}$ phase of TBPA $(\times 200)$.

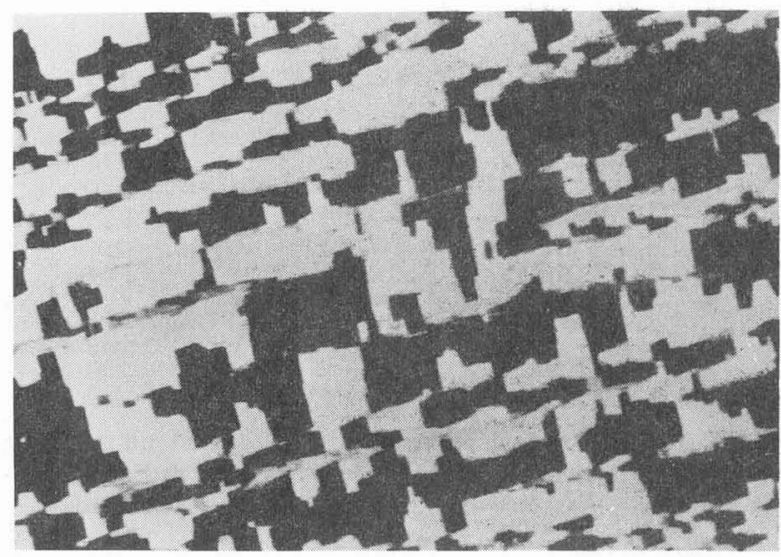

Plate 2. - The broken fan texture of the $S_{F}$ phase of TBPA $(\times 200)$.

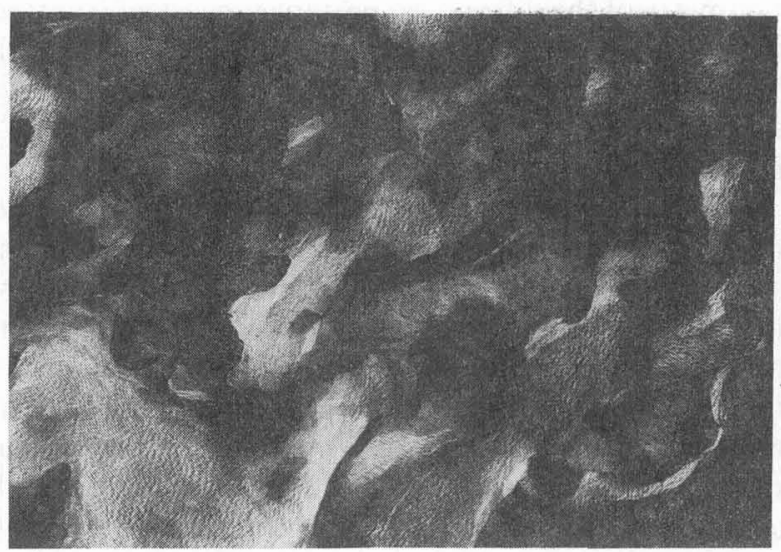

Plate 3, - The mosaic texture of the $S_{G}$ phase of TBPA $(\times 200)$.

of TBPA. The mosaic platelets are crossed with a fine zig-zag pattern. Plate 4 shows the broken fan and mosaic textures of the $S_{G}$ phase of TBPA.

Plate 5 shows the schlieren texture of the $S_{F}$ phase of $8 \mathrm{OSF}$; it is interesting to compare this with the corresponding texture for TBPA which is slightly more mosaic in character. Finally, plate 6 shows the mosaic texture of the $S_{G}$ phase of 8OSF. The mosaic platelets are crossed with a number of parallel lines

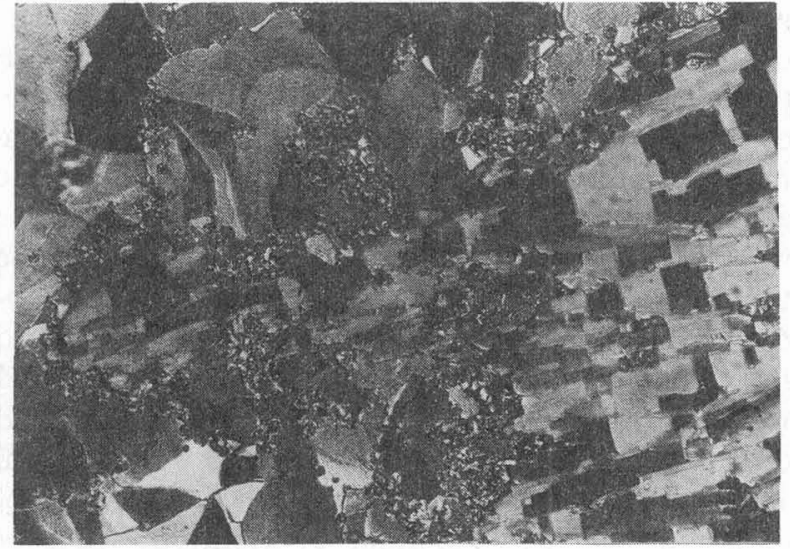

Plate 4. - The mosaic and broken fan textures of the $S_{G}$ phase of TBPA $(\times 200)$.

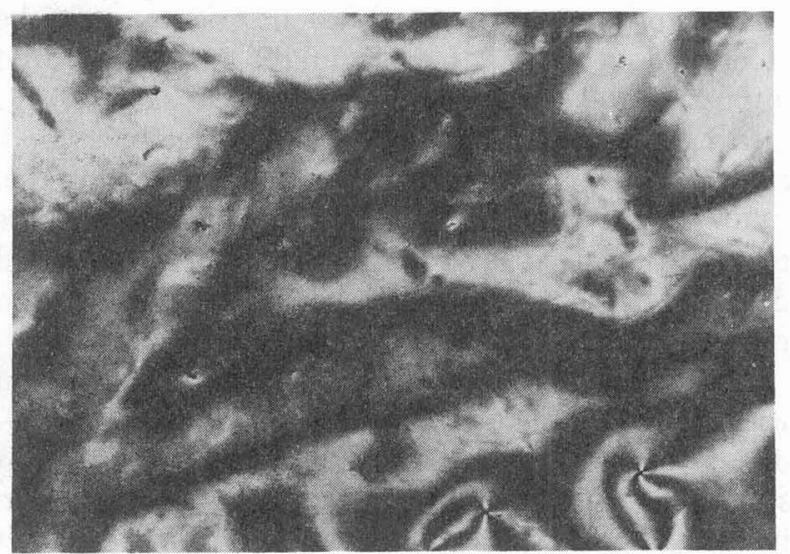

Plate 5, - The schlieren texture of the $S_{F}$ phase of 8 OSF $(\times 200)$.

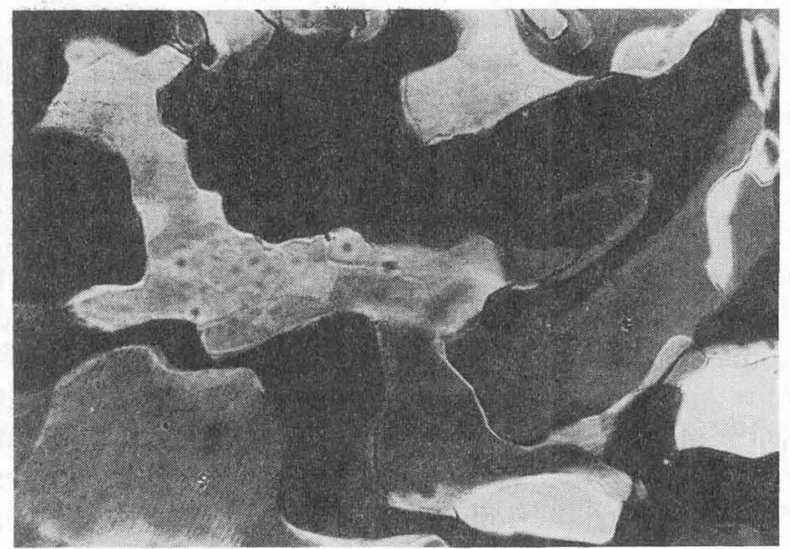

Plate 6. - The mosaic texture of the $S_{G}$ phase of TBPA $(\times 200)$.

in contrast with those shown by TBPA. Thus, these microscopic textures show slight differences in the phase types exhibited.

2. X-ray diffraction studies. -2.1 EXPERIMENTAL. - The samples were contained in Lindemann glass capillary tubes $(1.0 \mathrm{~mm}$ diameter $)$. By cooling the samples slowly $(\sim 1 \mathrm{~h})$ from the isotropic liquid to the $S_{A}$ phase in a strong magnetic field $(\sim 2$ tesla) 
we were able to obtain good monodomain samples. On cooling these to the $S_{C}$ and $S_{F}$ phases either with the field direction unchanged or without a field the orientation of the layers remained unchanged but the molecular tilt was randomly distributed around the azimuthal angle, relative to the layer normal. These will be called the partially aligned samples. For these tilted phases attempts were made to obtain monodomain samples by cooling the monodomain $S_{A}$ to the $S_{C}$ and $S_{F}$ phases with the magnetic field tilted with respect to the layer normal while keeping the field direction perpendicular to the incident X-ray beam. This resulted in specimens which, while not true monodomains, had an azimuthal distribution width of $\sim \pm 30^{\circ}$. Nevertheless, we will refer to these as the monodomain samples. On cooling either type of sample through the $S_{F}$ phase the quality of the alignment deteriorated with respect to the orientation of the layers and on cooling to the $S_{H}$ and $S_{G}$ phases it proved impossible to maintain a well aligned sample. We attribute this to change of tilt taking place by competative mechanisms in an increasingly viscous phase. The tilt angle may change by a tilting of the molecules relative to a fixed layer normal or by a longitudinal displacement of molecules with fixed orientation giving a tilt of the layer normal.

Diffraction patterns were recorded in transmission with monochromatic $(\mathrm{Cu}-\mathrm{K} \alpha)$ X-radiation perpendicular to a flat plate film. Patterns were recorded at several temperatures within each phase in order to investigate the variation of layer spacing and tilt angle with temperature. Powder patterns were also obtained for the $S_{H}$ phases of the two substances and preliminary data on the $S_{G}$ phases.

3. Results. - LAYER SPACINGS. - The variation of layer spacing (d) with temperature is presented graphically in figure 1 . We may define a tilt angle $\beta_{\mathrm{t}}$ as

$$
\beta_{\mathrm{t}}=\cos ^{-1} d / l
$$

where $l$ is the calculated molecular length in the most extended conformation. This definition is of course somewhat arbitrary in that it includes any changes of internal confirmation but is often in good agreement with more direct measurements [4]. The apparent tilt angle in the $S_{A}$ phase is simply a result of the fluctuation of the long axes of the molecules relative to the director, and the apparent tilt observed is consistent with $\left\langle P_{2}\right\rangle \sim 0.8-0.9$ which is reasonable for an $S_{A}$ phase.

4. The $\mathbf{S}_{\mathrm{F}}$ phase of TBPA. - Figure $2 b$ and $2 c$ shows the X-ray diffraction patterns obtained from the partially aligned and monodomain samples respectively. Two orders of Bragg reflections due to the smectic layers are visible which suggests an rms displacement of the molecules normal to the layers (along $c^{*}$ ) of $\left\langle Z^{2}\right\rangle^{1 / 2} \sim 4 \AA$. For the partially aligned sample, four diffraction maxima at high angle

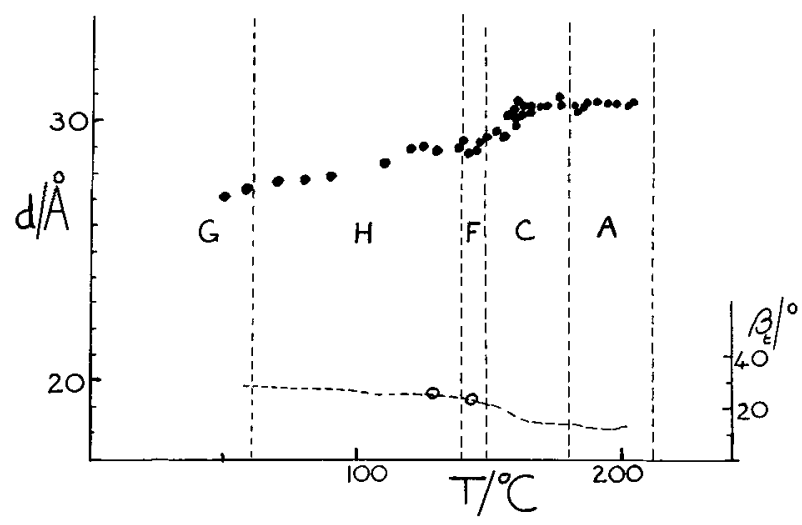

a)

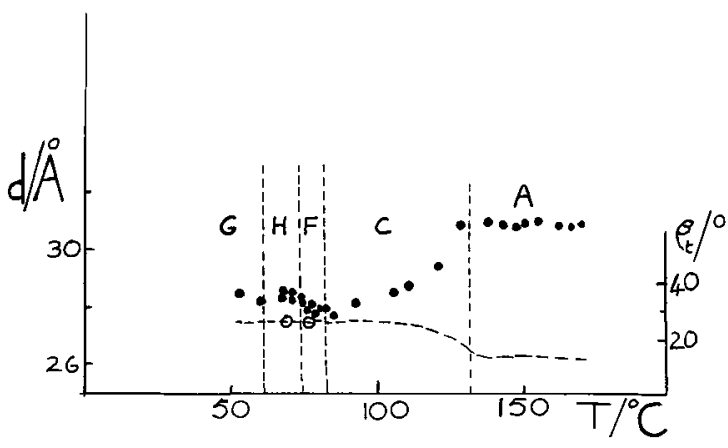

b)

FIG. 1. - The variation of layer spacing $(d)$ and tilt angle $\left(\beta_{\mathrm{t}}\right)$ with temperature for $(a)$ TBPA and (b) 8OSF. $d$, Experimental data ; - - $-\beta_{\mathrm{t}}=\cos ^{-1} d / l ; O, \beta_{\mathrm{t}}$ directly measured.

(two for the monodomain sample) are observed, indicating ordering within the smectic layers. The symmetry of the diffraction pattern suggest a $C$ centred unit cell with overall monoclinic symmetry, but with hexagonal packing of the molecules. Figure 3 shows diagrammatically the real and reciprocal lattices giving rise to the observed diffraction pattern.

The reciprocal lattice net is tilted about $\underline{b}^{*}$ and disordered about the layer normal $\left(c^{*}\right)$. The intersection of the reciprocal lattice points with the sphere of reflection then gives rise to the four reflections observed at high angle. That all the reflections lie on an arc indicates that within the accuracy of measurement the hexagon formed by the packing of the molecules is regular. The direction of tilt is the same as that observed for TBBA [5]. The hexagonal packing is confirmed by the symmetry of the diffraction pattern obtained with the X-ray beam perpendicular to the layers (Fig. 2e). The asymmetric intensities and appearance of the pattern arises from the $\left(23^{\circ}\right)$ tilt of the hexagon relative to the beam.

The tilt angle can be measured directly from the diffraction patterns like those of figure $2 c$. The result is included in figure 1 and it is clear that the value obtained indirectly from the layer spacings is close to the true tilt angle so that the temperature dependence of the tilt derived from the layer spacings is meaningful. 

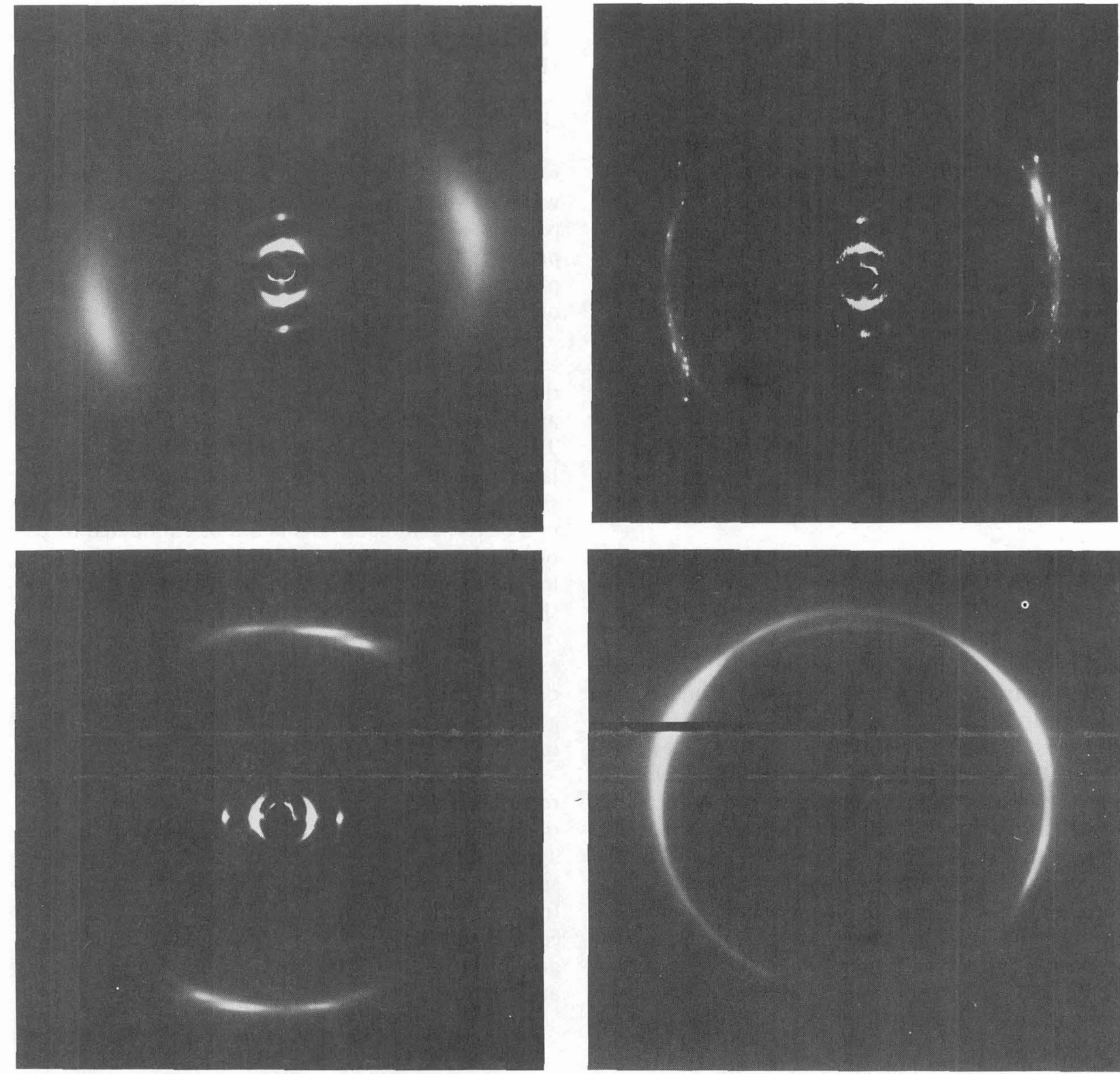

Fig. 2. - X-ray diffraction photographs of the $\mathrm{S}_{\mathrm{C}}(a), \mathrm{S}_{\mathrm{F}}(b)$ and $(c)$ and $\mathrm{S}_{\mathrm{H}}(d)$ phases of TBPA, (X-ray beam parallel to the smectic layers); (e) $\mathrm{S}_{\mathrm{F}}$ phase (X-ray beam perpendicular to the smectic layers)

The parameters for the $C$ centred monoclinic cell at $146^{\circ} \mathrm{C}$ are :

$$
\begin{aligned}
& \underline{a}=9.8 \AA \\
& \underline{b}=5.2 \AA \\
& \underline{c}=31.8 \AA \\
& \underline{\beta}=113^{\circ} .
\end{aligned}
$$

5. The shape of the diffraction spots. - The diffraction maxima at high angle in the $S_{F}$ phase are in the form of short bars whose width gives a measure of the correlation length for packing in the layers. Although the diffraction spots are broadened relative to those 

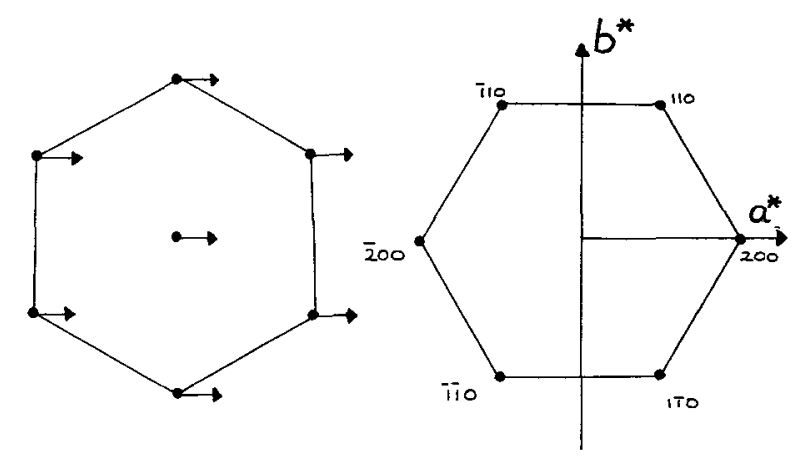

(a)

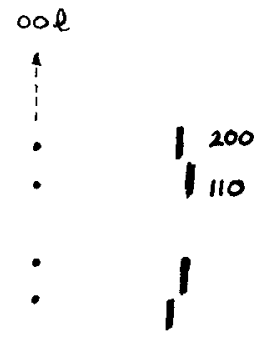

FIG. 3. - The real (a) and reciprocal (b) lattice, showing the hexagonal packing of molecules and the direction of tilt for TBPA.

for an $S_{H}$ or $S_{G}$ phase (or a crystal) they are considerably sharper than in the $S_{C}$ (or $S_{A}$ ) phases (see Fig. $2 a$ ) and the width suggests a correlation length within the layers of about 20 to 30 molecules (100$150 \AA$ ). The length of the bar is approximately equal to $c^{*}$ giving a correlation length for the $(h k o)$ planes normal to the layers of about one layer. Correlation between the layers is thus poor in the sense that the hexagonal nets have shift distortions between layers. However, the fact that a monodomain is obtained (tilts all in the same direction) shows that the hexagonal symmetry is preserved through the bulk of the sample (see Fig. 2e) and that the layers are therefore not free to rotate relative to each other.

The $\mathrm{S}_{\mathrm{H}}$ Phase. - Figure $2 d$ shows the diffraction pattern obtained from a partially aligned $S_{H}$ sample of TBPA. The symmetry is the same as that of the $S_{F}$ phase but with increased long range order as shown by the sharp diffraction spots. Upper and lower layer reflections are visible about the 200 and 110 reflexions. The quality of the photographs obtained show that the alignment deteriorates on cooling through the $S_{\mathrm{F}}$ to the $\mathrm{S}_{\mathrm{H}}$ and we have as yet been unable to obtain a well aligned $S_{H}$ specimen.

The powder photograph of the $S_{H}$ phase of TBPA shows five rings at high angle and these can be indexed as for the $S_{H}$ phase of TBBA [5].

The structure can thus be identified as a $C$ centred monoclinic cell with essentially regular hexagonal packing of the molecules and unit cell parameters at $130^{\circ} \mathrm{C}$ are

$$
\underline{a}=9.5 \AA ; b=5.6 \AA ; C=31.2 \AA ; \beta=115^{\circ} \text {. }
$$

As for the $S_{F}$ phase the directly measured tilt angle is the same as that obtained from the layer spacings (Fig. 1). The smectic layers themselves are only slightly more well defined than for the $\mathrm{S}_{\mathrm{F}}$ phase, with $\left\langle Z^{2}\right\rangle^{1 / 2} \sim 3.5 \AA$.

The $S_{G}$ Phase. - On cooling to the $S_{G}$ phase there was increased deterioration in sample alignment and we were unable to obtain good clear diffraction patterns of the $S_{G}$ phase. Nevertheless it seems highly probable from the results obtained so far that this phase is quite analogous to the $S_{G}$ phase (phase VI) of TBBA.

The $\mathrm{S}_{\mathrm{F}}$ Phase of 8OSF. - Figure $4 b$ and $c$ show the diffraction patterns obtained from partially aligned and monodomain samples of the $S_{F}$ phase of $8 O S F$. Three orders of Bragg reflections from the smectic layers are clearly visible. The appearance of a double spot in some cases was found to be attributable to use of a coarse collimator and could be eliminated by use of a finely collimated X-ray beam (with of course some loss of intensity). The diffraction pattern at high angle shows three diffuse maxima, in contrast to the four observed for the $S_{F}$ phase of TBPA, and can again be explained in terms of hexagonal packing in a plane perpendicular to the molecules. In this case the hexagonal net must be tilted differently to that of TBPA in order to explain the observed pattern.

This is shown in figure 5 by considering the real and reciprocal lattice nets. Again the tilt angle can be obtained directly from the photographs and is in agreement with the values calculated from the layer spacings so that the temperature dependence obtained from the latter will be meaningful. The cell is again $C$ centred monoclinic, but with the tilt direction orthogonal to that for TBPA and the unit cell parameters at $78^{\circ} \mathrm{C}$ are

$$
\underline{a}=5.2 \AA ; \underline{b}=9.1 \AA ; \underline{c}=30.6 \AA ; \beta=112 .
$$

The smectic layer distribution is slightly sharper than that for TBPA with $\left\langle Z^{2}\right\rangle^{1 / 2} \sim 3.3 \AA$.

As for TBPA the correlation of the hexagonal packing within a layer extends over about 20-30 molecules while the correlation between layers is very weak indeed amounting to only about 1 layer.

The $S_{H}$ Phase. - In the $S_{H}$ diffraction photographs four orders of Bragg reflections from the smectic layers are visible indicating well defined layers with $\left\langle Z^{2}\right\rangle^{1 / 2} \sim 2.5 \AA$. At high angle there are essentially the same features as in the $S_{F}$ phase but with the addition of two extra reflections (Fig. 4d) arising from upper and lower levels of the hexagonal reciprocal lattice net. The powder pattern of the $S_{H}$ phase shows only three rings (cf. 5 for TBPA) which can be indexed in terms of the observed tilt. The parameters of the $C$ centred monoclinic cell at $65^{\circ} \mathrm{C}$ are then :

$$
\underline{a}=5.2 \AA ; \underline{b}=9.0 \AA ; \underline{c}=31.0 \AA ; \beta=114^{\circ} .
$$


a)
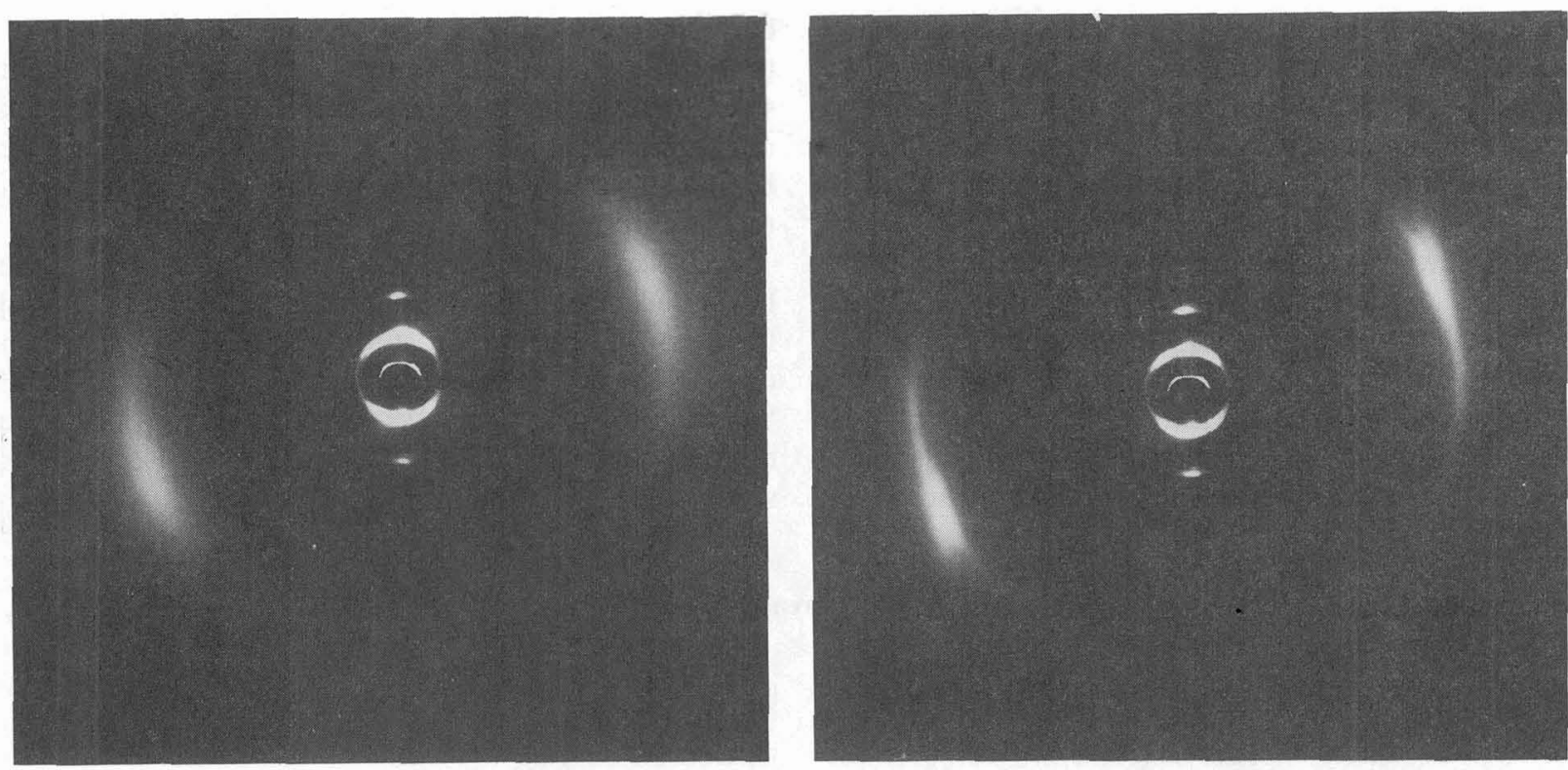

c)

b)
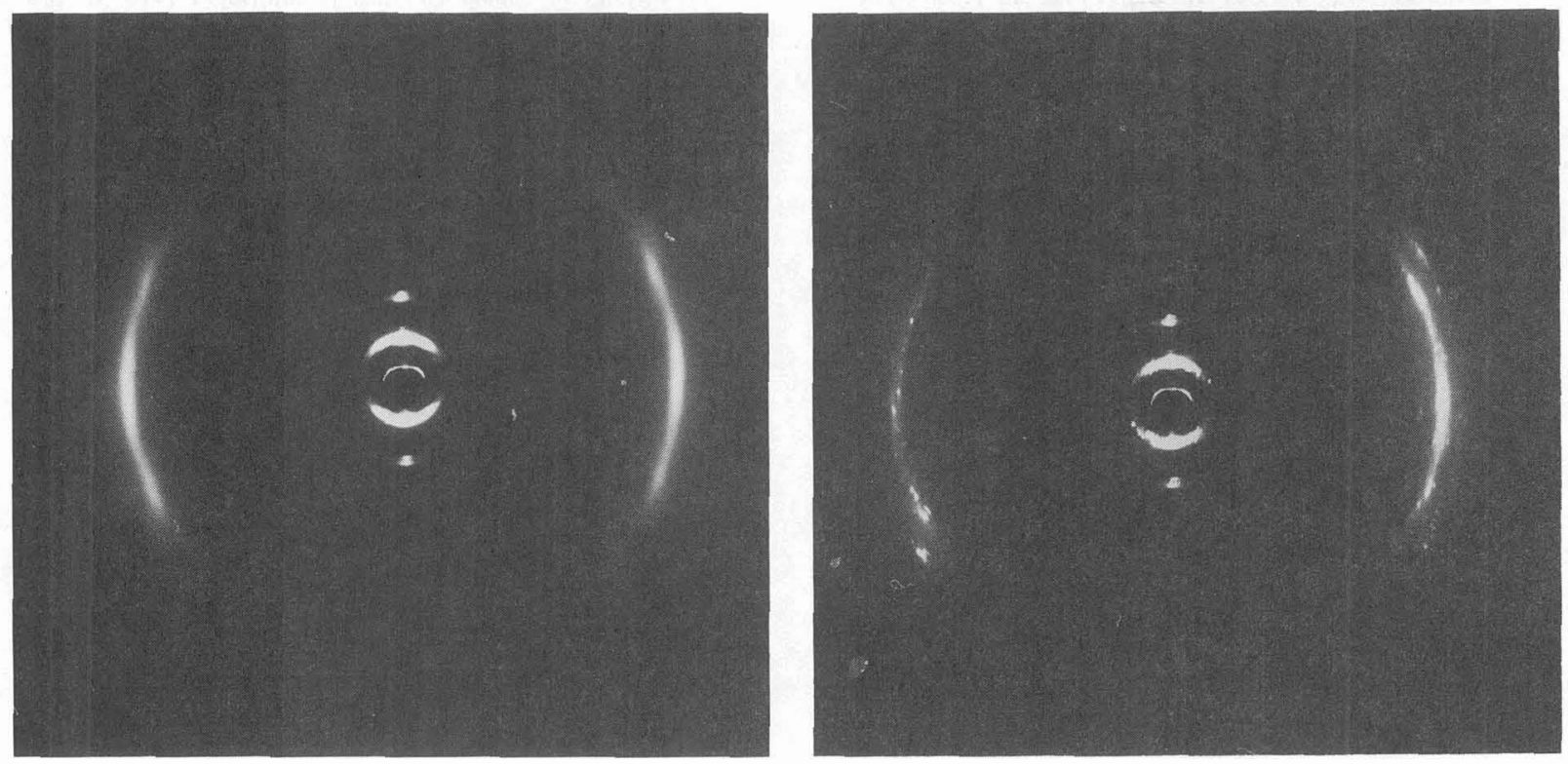

d)

FIG. 4. - X-ray diffraction photographs of the $\mathrm{S}_{\mathrm{C}}(a), \mathrm{S}_{\mathrm{F}}(b)$ and $(c), \mathrm{S}_{\mathrm{H}}(d)$ phases of $80 \mathrm{OSF}$, (X-ray beam parallel to the smectic layers).

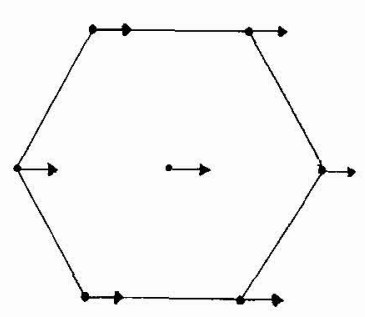

(a)

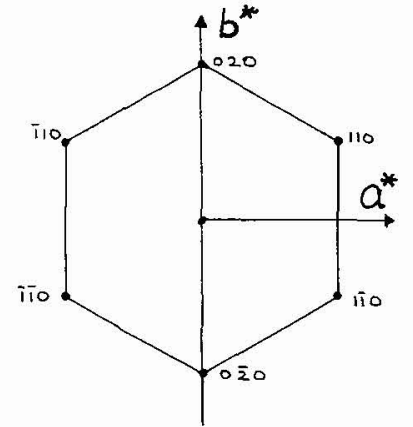

(b)

FIG. 5. - The real (a) and reciprocal (b) lattice, showing the hexagonal packing of molecules and the direction of tilt for $8 \mathrm{OSF}$. 4

The tilt angle from the layer spacings is again in good agreement with that directly measured.

The $S_{G}$ Phase, - Again deterioration in sample alignment made it impossible to obtain good diffraction patterns of the $S_{G}$ phase but it is clear that the tilt direction remains the same as in the $S_{F}$ and $S_{H}$ phases but increases in magnitude to about $25^{\circ}$.

6. Conclusions. - The diffraction studies of the $S_{F}$ phases of TBPA and 8OSF have enabled the 
structures of these phases to be deduced for the first time. In fact the symmetries of the phases of the two materials are slightly different : both have a regular hexagonal packing of molecules of limited $(\sim 30$ molecular diameters) correlation length tilted relative to the layer normal. However, the hexagon in TBPA is tilted towards an edge while for 8OSF the tilt is towards a corner. The same two symmetries of tilt are found in both $S_{H}$ and $S_{G}$ phases of the respective materials although more work remains to be done on the detailed characterisation of the $S_{G}$ phases. It is interesting to note that the identically labelled smectic phases of these compounds appear to be completely miscible indicating that they are the same thermodynamic phase while having distinctly different structures. This may well be a consequence of the lack of long range order in the layers. It would therefore be interesting to study the structures of mixtures of these compounds.

Another example of an $S_{H}$ phase tilted towards the hexagonal apex has been inferred by Doucet et al. [6] from powder diffraction studies of HOBACPC which is a ferroelectric smectic. This material like $8 \mathrm{OSF}$ has a chiral centre but we examined only a racemic mixture while for HOBACPC a simple isomer was studied.

\section{References}

[1] Goodby, J. W. and Gray, G. W., to be published in J. Physique.

[2] Demus, D., Diele, S., Klapperstück, M., Link, V. and ZaschKe, H., Mol. Cryst. Liq. Cryst. 15 (1971) 161.

[3] Goodby, J. W., Gray, G. W. and Mosley, A., Mol. Cryst. Liq. Cryst. Lett. 41 (1978) 183.
[4] Doucet, J., Levelut, A. M., Lambert, M., Mol. Cryst. Liq. Cryst. 24 (1973) 317.

[5] DouceT, J., Nato Advanced Study Institute on Molecular Physics of Liquid Crystals (Cambridge) 1977. In press.

[6] Doucet, J., Keller, P., Levelut, A. M., Porquet, P., J. Physique 39 (1978) 548. 